PAPER • OPEN ACCESS

\title{
Bouncing in an oscillatory gravitational field
}

To cite this article: J L Martin and P Strange 2021 Eur. J. Phys. 42045409

View the article online for updates and enhancements.

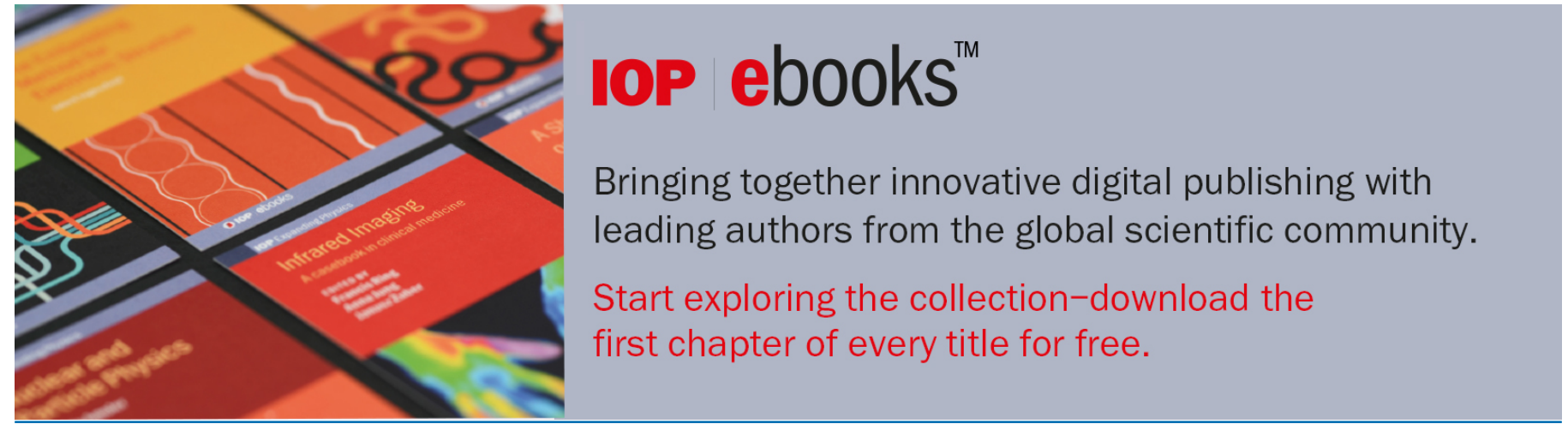

This content was downloaded from IP address 94.12 .187 .99 on 07/06/2021 at 09:58 


\title{
Bouncing in an oscillatory gravitational field
}

\author{
J L Martin and P Strange* \\ School of Physical Sciences, University of Kent, Canterbury, Kent, CT2 7NH, United \\ Kingdom \\ E-mail: P.Strange@kent.c.uk
}

Received 3 February 2021, revised 26 March 2021

Accepted for publication 16 April 2021

Published 3 June 2021

\begin{abstract}
We apply the time-dependent supersymmetry methods of Bagrov, Samsonov and Shekoyan to the Schrödinger equation for a quantum bouncer. A new family of potentials, that correspond to the linear gravitational potential with a small oscillatory component superimposed upon it, is produced. Both the frequency and amplitude of the oscillatory part can be controlled and the corresponding eigenfunctions are found. These solutions are explored and basic observables are calculated. In particular we examine how motion in such a potential can be distinguished from motion in a linear gravitational field. We also point out some pedagogical aspects of this project.
\end{abstract}

Keywords: quantum, bouncing, supersymmetry

(Some figures may appear in colour only in the online journal)

\section{Introduction}

Bouncing on a surface in the way we commonly understand it is very much a classical mechanical phenomena. It is essentially a collision between two objects in which energy and momentum are conserved. At each bounce the momentum of the bouncer is reversed and the momentum difference is transferred to the surface. If the surface is ground level on the Earth the potential energy of the bouncer is accurately written as $V(z)=m g z$ where $z$ is height above the ground. At each bounce the momentum transfer to the Earth is negligible and is absorbed into the atoms and molecules close to the point of contact between the bouncer and the Earth. The height attained by the bouncer depends on the elastic properties of the materials that make

*Author to whom any correspondence should be addressed.

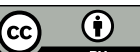

Original content from this work may be used under the terms of the Creative Commons Attribution 4.0 licence. Any further distribution of this work must maintain attribution to the author(s) and the title of the work, journal citation and DOI. 
up both the surface and the bouncer, so for example, a rubber ball will bounce on a concrete surface while a ball made of lead will not. To create a quantum mechanical description of this sort of bouncing would be a very demanding project indeed.

The Schrödinger equation forms the foundation of non-relativistic quantum mechanics. It has only very few physically meaningful exact solutions. Most of the familiar models in quantum theory are steady-state solutions which means that the space and time dependence of the problem can be separated. These, such as the harmonic oscillator and the one-electron atom, form the basis of much of our understanding of the physics of nature.

One such model that has been extensively examined is known as the quantum bouncer, where the potential $V(z)=m g z$ is linear in the height above a hard surface, which can be solved exactly. The properties this model exhibits bear little resemblance to classical bouncing. A number of classic texts describe the quantum bouncer in pedagogical detail (see references $[1,2]$ for example). The properties of the solutions were discussed by Gibbs [3]. Later a more systematic investigation was reported by Gea-Banacloche [4] who also provided a clear discussion of how to obtain the classical limit of quantum bouncing. However in general the particle executes the harmonic motion typical of interfering wavepackets and the expectation value of position never gets close to the surface. Gea-Banacloche's work has been extended in a number of directions [5-7]. Good discussions of various aspects of the quantum bouncer are given in references $[8,9]$. Experimentally there has been a long debate concerning the observation of bound quantum states in a gravitational field [10-14].

Non-stationary supersymmetric quantum theory has been derived [15-17] and extended [18] and provides a strategy for finding new solutions of the time-dependent Schrödinger equation if we know one solution. This work is a natural extension of the time independent supersymmetric methods discussed in very readable form by Cooper et al [19]. It is a very powerful technique but its implementation has been limited so far, Bagrov et al performed a number of examples in their papers [15-17] deriving the method, although these contain little physical interpretation of the results, and Zelaya and Rosas-Ortiz [20] have found interesting new potentials starting from the harmonic oscillator, while Rasinskaite and Strange [21] have used the method to describe quantum surfing.

In this paper we have started with the linear gravitational potential and performed two supersymmetry transformations on it. This has led us to be able to introduce a family of potentials in the same spirit as those introduced by Zelaya and Rosas-Ortiz [20]. These new potentials are the original linear potential with a small oscillatory component superimposed upon it where we can control both the amplitude and frequency of the oscillatory part. This enables us to examine observables and address the question of how we would know if the gravitational field of the Earth was not simply linearly proportional to height above the Earth, but included a small oscillatory component. The work reported here is the result of a student project and demonstrates how interesting results can be generated using non-stationary supersymmetry theory as the basis of such a project [21].

This paper is organised as follows. In section 2 we review the salient results for the standard quantum bouncer. Supersymmetry theory is well described in the standard literature, so we do not reproduce that here, instead we simply provide sufficient information which, in tandem with the original literature, can be used to reproduce our results if required. In section 4 we report a new family of exactly soluble potentials, based on the quantum bouncer, derived by employing supersymmetry techniques. We present the eigenfunctions, eigenvalues and expectation values of observables for the oscillatory gravitational field case and compare them with the results for the linear potential. This enables us to discuss how to distinguish these potentials experimentally. In section 5 we discuss the mechanism for bouncing and point out the role of 
a purely quantum mechanical force. Finally we draw a few key conclusions about this work. Throughout this paper we work in units in which $m=1 / 2, g=1$ and $\hbar=1$.

\section{The quantum bouncer}

The standard bouncer involves a particle under the influence of gravity bouncing off a perfectly reflecting surface. In this section we provide a brief overview of the standard bouncer based on the paper of Gea-Banacloche [4] If we consider the $z$-axis as being in the vertical direction

$$
\begin{aligned}
V(z) & =m g z \quad z>0 \\
& =\infty \quad z<0
\end{aligned}
$$

The time-dependent Schrödinger equation is

$$
-\frac{\hbar^{2}}{2 m} \frac{\partial^{2} \Psi_{n}(z, t)}{\partial z^{2}}+m g z \Psi_{n}(z, t)=\mathrm{i} \hbar \frac{\partial \Psi_{n}(z, t)}{\partial t}
$$

and we have the boundary condition

$$
\Psi_{n}(0, t)=0
$$

The time-dependent Schrödinger equation separates $\Psi_{n}(z, t)=\psi_{n}(z) \Phi(t)=\psi_{n}(z) \exp$ $\left(-\mathrm{i} \Sigma_{n} t / \hbar\right)$ with

$$
-\frac{\hbar^{2}}{2 m} \frac{\partial^{2} \psi_{n}(z)}{\partial z^{2}}+m g z \psi_{n}(z)=\Sigma_{n} \psi_{n}(z)
$$

Following Gea-Banacloche [4] we introduce a gravitational length $l_{\mathrm{g}}$ and time $t_{\mathrm{g}}$

$$
l_{\mathrm{g}}=\left(\frac{\hbar^{2}}{2 g m^{2}}\right)^{1 / 3} \quad t_{\mathrm{g}}=\left(\frac{2 \hbar}{m g^{2}}\right)^{1 / 3} \eta=\left(\frac{2}{\hbar^{2} m g^{2}}\right)^{1 / 3}
$$

and then we introduce the dimensionless quantities

$$
x=z / l_{\mathrm{g}} \quad E_{n}=\eta \Sigma_{n} .
$$

With this change of variables the Schrödinger equation becomes

$$
\frac{\partial^{2} \psi_{n}(x)}{\partial x^{2}}-\left(x-E_{n}\right) \psi_{n}(x)=0
$$

This is the equation defining an Airy function [22]. As the potential is not bounded above the Bi solution [22], which goes to infinity for large values of its argument, is not physical and so

$$
\psi_{n}(x)=A_{n} \operatorname{Ai}\left(x-E_{n}\right),
$$

where $E_{n}$ is chosen so that equation (3) is satisfied and $A_{n}$ is the normalisation. For the wellknown solutions of the Schrödinger equation the eigenfunctions form a family of solutions. In this case the solutions for different values of $n$ are all the same function, $n$ forms a label for the particular zero of the Airy function that we use to terminate the eigenfunction at $z=0$. A good approximation for the normalisation is

$$
A_{n}=\left(\frac{2 \pi^{2}}{3(n-1 / 4)}\right)^{1 / 6}
$$


however we have chosen to determine it numerically. Quantum mechanical bouncing is found to be very different to classical bouncing as it evolves due to quantum mechanical interference and the expectation value of position always stays well above the bouncing surface. By taking a Gaussian distribution of these solutions Gea-Banacloche [4] has explored the properties of this bouncer including exhibiting the classical limit and displaying quantum collapse and revival phenomena.

\section{Supersymmetry}

This method has been well-described in a number of publications [15-18] and is a natural extension of the stationary supersymmetry theory [19]. Previous applications include to the harmonic oscillator [20] and to describe quantum surfing [21].

In supersymmetry theory we start with a potential with its associated Hamiltonian and two distinct eigenfunctions of that Hamiltonian. A transformation is applied that provides us with a new potential and the associated eigenfunctions that solve a new Schrödinger equation. The new potential and eigenfunctions can then be used as input to a further transformation, and so on, so a hierarchy of solutions of the Schrödinger equation can be generated. For the purposes of this project it was necessary to perform two such transformations.

For the first transformation our initial potential and eigenfunctions are given by equations (1) and (8) for two different values of $\Sigma_{n}$ which we call $w_{\alpha}$ and $w_{\beta}$. It is not necessary at this stage to choose $w_{\alpha}$ and $w_{\beta}$ as solutions that satisfy equation (3). These are the input to a first supersymmetry transformation and yield a potential which depends on $w_{a}$ and $z$ and eigenfunctions

$$
\xi_{\alpha, \beta}(z)=B_{\beta}\left(-\frac{\left.\operatorname{Ai}\left(z / l_{\mathrm{g}}-\eta w_{\beta}\right) \mathrm{Ai}^{\prime}\left(z / l_{\mathrm{g}}-\eta w_{\alpha}\right)\right)}{\operatorname{Ai}\left(z / l_{\mathrm{g}}-\eta w_{\alpha}\right)}+\mathrm{Ai}^{\prime}\left(z / l_{\mathrm{g}}-\eta w_{\beta}\right)\right) \mathrm{e}^{\mathrm{i} w_{\beta} t / \hbar},
$$

where $B_{\beta}$ is the normalisation, although it is not necessary to normalise at this stage. The prime on the Airy function represents differentiation with respect to its argument.

For the second transformation we use the potential output from the first transformation and two eigenfunctions of the form of equation (10), $\xi_{a, b}(z)$ and $\xi_{a, c}(z)$. This yields a potential which depends on $w_{a}, w_{b}$ and $z$ and eigenfunctions that depend on $w_{a}, w_{b}, w_{c}$ and $z$. Next we choose $w_{a}$ and $w_{b}$ arbitrarily and that fixes the potential. Then the different permitted eigenfunctions of the Hamiltonian associated with that potential are determined by the values of $w_{c}$ that cause the eigenfunctions to satisfy equation (3). We label these $w_{n}$ with $1 \leqslant n<\infty$ and they define the permitted energy spectrum of the new Hamiltonian.

\section{Bouncer in an oscillatory gravitational field}

Any further intervening formulae in the supersymmetry theory are too long to write down, but we finally obtain the following. If we define

$$
\begin{aligned}
V_{1}(z)= & \left(m^{2} g x+\eta \hbar^{2}\left(w_{a}-w_{b}\right) / l_{\mathrm{g}}^{2}\right) \mathrm{Ai}^{2}\left(z / l_{\mathrm{g}}-\eta w_{b}\right) \mathrm{Ai}^{\prime 2}\left(z / l_{\mathrm{g}}-\eta w_{a}\right) \\
V_{2}(z)= & 2 m^{2} g x \operatorname{Ai}\left(z / l_{\mathrm{g}}-\eta w_{a}\right) \operatorname{Ai}^{\prime}\left(z / l_{\mathrm{g}}-\eta w_{a}\right) \operatorname{Ai}\left(z / l_{\mathrm{g}}-\eta w_{b}\right) \\
& \times \operatorname{Ai}^{\prime}\left(z / l_{\mathrm{g}}-\eta w_{b}\right) \\
V_{3}(z)= & \left(w_{a}-w_{b}\right)^{2} \eta^{2} \hbar^{2} \operatorname{Ai}^{2}\left(z / l_{\mathrm{g}}-\eta w_{a}\right) \mathrm{Ai}^{2}\left(z / l_{\mathrm{g}}-\eta w_{b}\right) / l_{\mathrm{g}}^{2} \\
V_{4}(z)= & \left(m^{2} g z-\left(w_{a}-w_{b}\right) \eta \hbar^{2} / l_{\mathrm{g}}^{2}\right) \mathrm{Ai}^{2}\left(z / l_{\mathrm{g}}-\eta w_{a}\right) \mathrm{Ai}^{\prime 2}\left(z / l_{\mathrm{g}}-\eta w_{b}\right)
\end{aligned}
$$



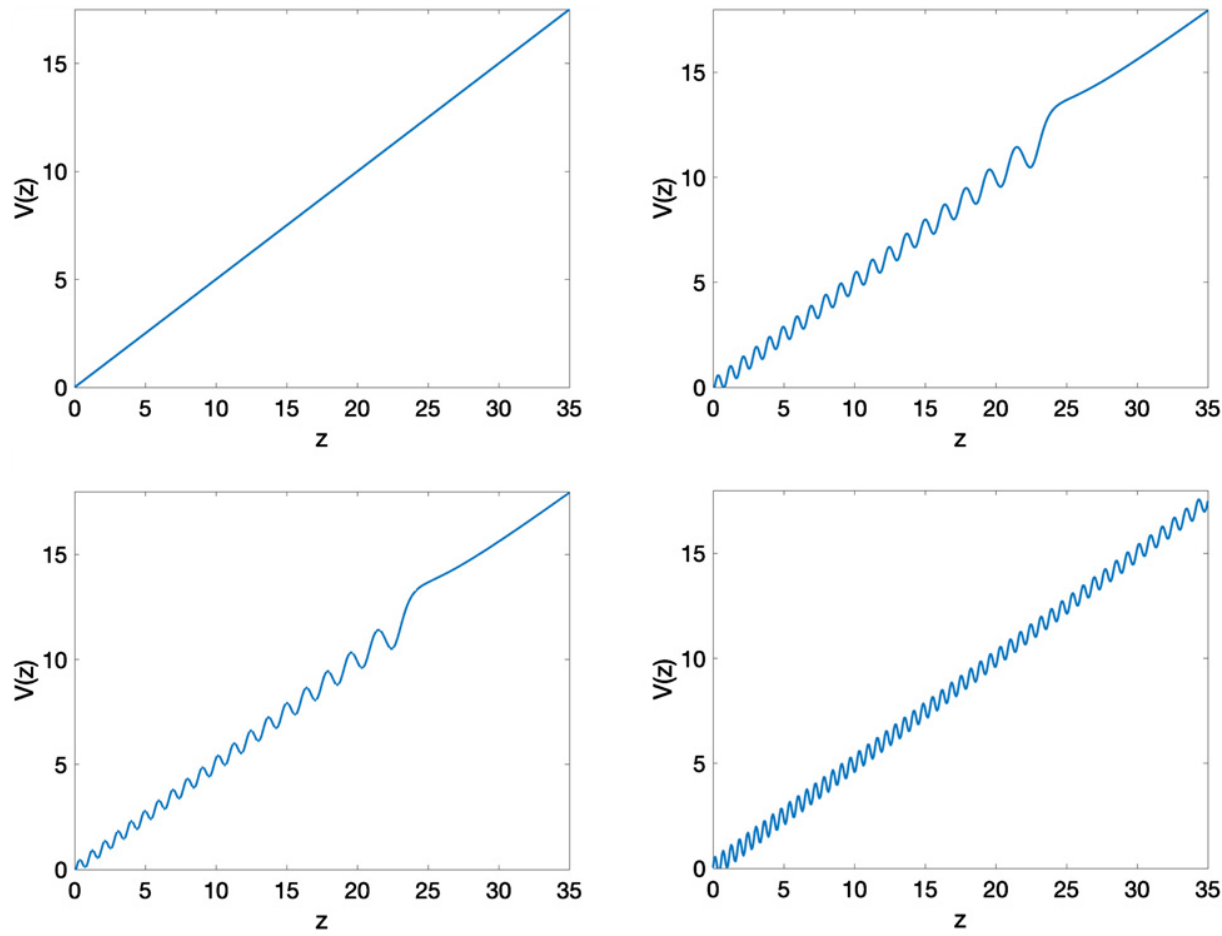

Figure 1. Top left: the linear potential of equation (1) for the standard quantum bouncer Top right: the potential of equation (11) with $w_{a}=13.10$ and $w_{b}=12.90$. Bottom left: the potential of equation (11) with $w_{a}=13.01$ and $w_{b}=12.99$. Bottom right: the potential of equation (11) with $w_{a}=30.10$ and $w_{b}=29.90$ (this becomes linear at a much higher value of $z$ ).

then the theory yields the following potential

$$
V(z)=\frac{V_{1}(z)-V_{2}(z)+V_{3}(z)+V_{4}(z)}{\left(m\left(\operatorname{Ai}\left(z / l_{\mathrm{g}}-\eta w_{b}\right) \operatorname{Ai}^{\prime}\left(z / l_{\mathrm{g}}-\eta w_{a}\right)-\operatorname{Ai}\left(z / l_{\mathrm{g}}-\eta w_{a}\right) \mathrm{Ai}^{\prime}\left(z / l_{\mathrm{g}}-\eta w_{b}\right)\right)\right)},
$$

where $W\left(\operatorname{Ai}\left(z / l_{\mathrm{g}}-\eta w_{b}\right), \operatorname{Ai}\left(z / l_{\mathrm{g}}-\eta w_{a}\right)\right)$ is the Wronskian. Furthermore if we define

$$
\begin{aligned}
& \chi_{(1) n}(z)=\eta\left(\left(w_{n}-w_{a}\right) \operatorname{Ai}\left(z / l_{\mathrm{g}}-\eta w_{a}\right) \operatorname{Ai}\left(z / l_{\mathrm{g}}-\eta w_{n}\right) \operatorname{Ai}^{\prime}\left(z / l_{\mathrm{g}}-\eta w_{b}\right)\right) \\
& \chi_{(2) n}(z)=\left(w_{b}-w_{n}\right) \operatorname{Ai}\left(z / l_{\mathrm{g}}-\eta w_{b}\right) \operatorname{Ai}\left(z / l_{\mathrm{g}}-\eta w_{n}\right) \operatorname{Ai}^{\prime}\left(z / l_{\mathrm{g}}-\eta w_{1}\right) \\
& \left(\chi_{(3) n}(z)=\left(w_{a}-w_{b}\right) \operatorname{Ai}\left(z / l_{\mathrm{g}}-\eta w_{a}\right) \operatorname{Ai}\left(z / l_{\mathrm{g}}-\eta w_{b}\right) \operatorname{Ai}^{\prime}\left(z / l_{\mathrm{g}}-\eta w_{n}\right) / l_{\mathrm{g}}^{2}\right)
\end{aligned}
$$

then the eigenfunctions of the Schrödinger equation, $\phi_{n}(z)$, associated with the potential $V(z)$ are given by:

$$
\phi_{n}(z)=N_{n} \frac{\chi_{(1) n}(z)+\chi_{(2) n}(z)+\chi_{(3) n}(z)}{\operatorname{Ai}\left(z / l_{g}-\eta w_{b}\right) \operatorname{Ai}^{\prime}\left(z / l_{\mathrm{g}}-\eta w_{a}\right)-\operatorname{Ai}\left(z / l_{\mathrm{g}}-\eta w_{a}\right) \mathrm{Ai}^{\prime}\left(z / l_{\mathrm{g}}-\eta w_{b}\right)},
$$

where $N_{n}$ is the normalisation coefficient which we determine numerically. 

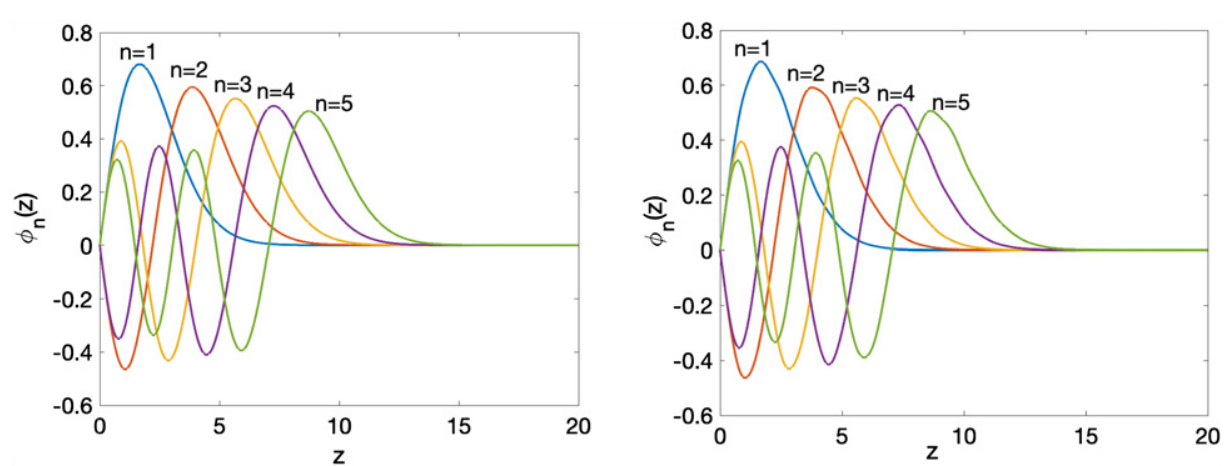

Figure 2. The lowest five eigenfunctions $\phi_{n}(z)$ for the quantum bouncer. Left: for the linear potential, equation (8), right: for the oscillatory potential with $w_{a}=13.1$ and $w_{b}=12.9$, equation (13).

The potential for the quantum bouncer is shown in figure 1. In the top left we have the usual linear potential for the standard bouncer given by equation (1). In the other diagrams we illustrate the effect of varying $w_{a}$ and $w_{b}$. We observe that an increase in these parameters decreases the wavelength of the oscillations, while bringing them closer together decreases the amplitude, particularly close to the 'ground' level. At high values of $z$ the potential becomes linear for all potentials, but we confine ourselves to cases where the top of the bounce is well below this linear regime unless otherwise stated. If $\left|w_{a}-w_{b}>\right| \gtrsim 0.3$ we find a singularity in the potential which, although interesting in itself, is not part of the current project. The potentials in figure 1 have an infinite potential below $z=0$. Above $z=0$ the potential generally increases from close to zero. and so $V \rightarrow \infty$ as $z \rightarrow \infty$. This means that the potential is confining and the particle is trapped. Therefore we expect some sort of oscillatory behaviour to occur.

Next we consider the Hamiltonian eigenfunctions for these potentials. The general case can be illustrated using $w_{a}=13.1$ and $w_{b}=12.9$ In figure 2 we show the lowest five eigenfunctions for the linear potential (left) and the oscillatory potential (right) for cases where $w_{n} \ll w_{b}$. We observe that there are minor differences, but that the eigenfunctions are really broadly similar. Any observables calculated from these eigenfunctions might differ in details, but would be qualitatively much the same. In figure 3 we show the eigenfunctions for $n=19,20,21$ which correspond to $w_{n}=12.4973,12.9377,13.3708$ for the linear potential and $w_{n}=12.4856,13.3927,13.8065$ for the oscillatory potential. Clearly at values of $w_{n}$ close to $w_{a}$ and $w_{b}$ the eigenfunctions are qualitatively different for the two potentials. In fact it appears that the oscillatory potential 'omits' an eigenvalue. In fact this is not the case. It is a known property of the supersymmetry theory that if we set $w_{n}$ equal to $w_{a}$ or $w_{b}$ the eigenfunction will be zero at all values of $z$. If we go to higher values of the quantum numbers the eigenfunctions become similar again.

A general wavepacket is defined as:

$$
\Phi(z, t)=\sum_{n=1}^{\infty} C_{n} \mathrm{e}^{-\mathrm{i} w_{n} t / \hbar} \phi_{n}(z) .
$$

We make the same assumption as Gea-Banacloche, that the starting condition is that of a Gaussian wave packet of the form:

$$
\Phi(z, 0)=\left(\frac{2}{\pi \sigma^{2}}\right)^{\frac{1}{4}} \mathrm{e}^{-\left(z-z_{0}\right)^{2} / \sigma^{2}}
$$



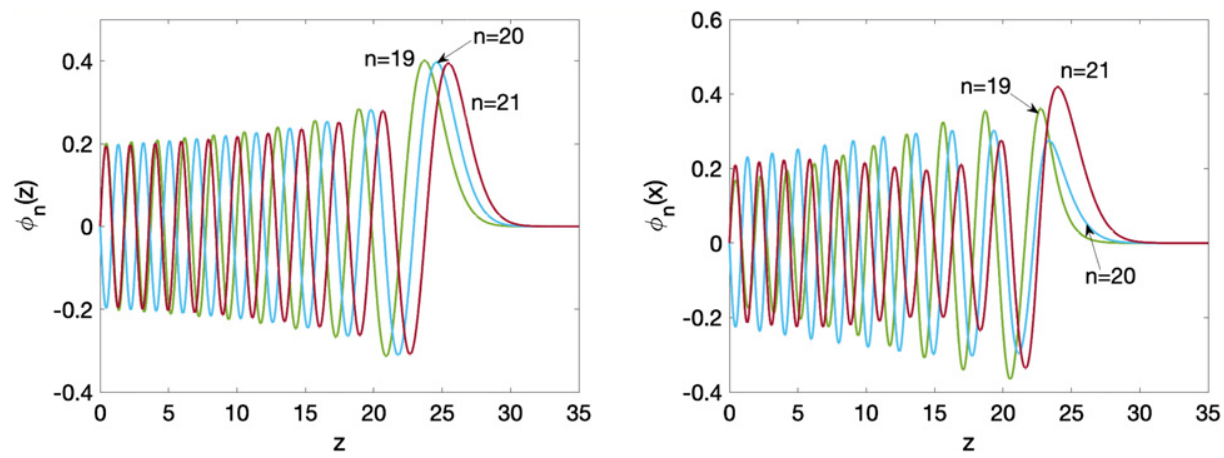

Figure 3. The eigenfunctions of a quantum bouncer for quantum numbers $n=19,20,21$. Left: for the linear potential, equation (8). Right: for the oscillatory potential with $w_{a}=13.1$ and $w_{b}=12.9$, equation (13). For these values of $n, w_{n}$ is in the range $12.0<w_{n}<14.0$.
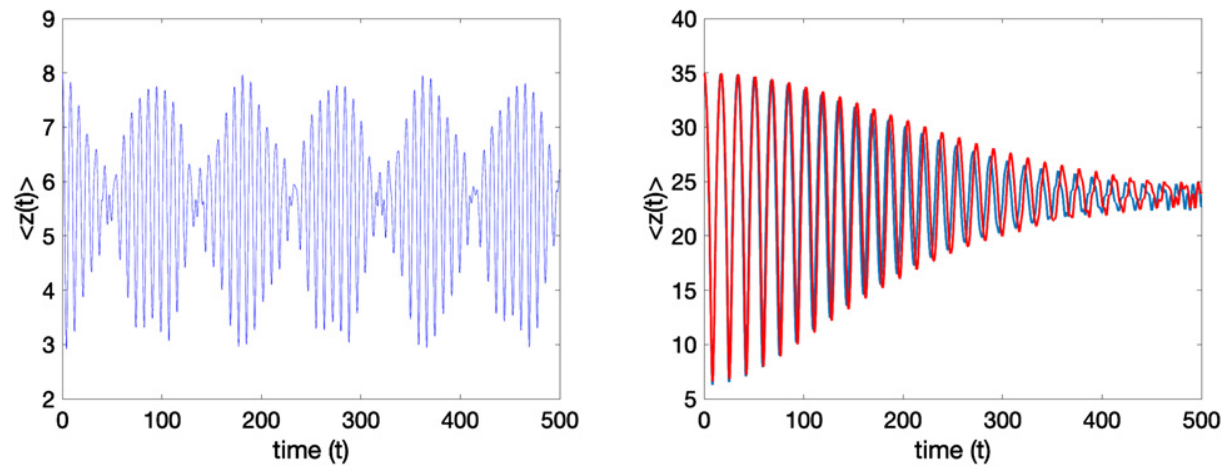

Figure 4. The expectation value of position as a function of time for the wavepacket described by equations (14) and (16). Left: $z_{0}=8$ and $\sigma=2.0$, here the results for both the oscillatory potential and the linear potential are overlaid and the results from the two potentials are indistinguishable on this scale. Right: $z_{0}=35$ and $\sigma=2.0$ the blue line is for the linear potential and the red line is for the oscillatory potential.

where $\sigma$ is a measure of the width of the wave packet and $z_{0}$ is the initial drop height. This gives the coefficients of the expansion, $C_{n}$, as:

$$
C_{n}=\left(\frac{2}{\pi \sigma^{2}}\right)^{\frac{1}{4}} \int_{0}^{\infty} \phi_{n}(z) \mathrm{e}^{-\left(z-z_{n}\right)^{2} / \sigma^{2}} \mathrm{~d} z
$$

With this initial condition we can calculate the evolution of the wavepacket in both potentials.

In figure 4 we show the expectation value of position above the ground as a function of time for the bouncer. On the left side the initial drop height is $z(0)=8$. This means that all the values of $w_{n}$ which contribute significantly to the wavefunction are lower than $w_{b}$. There are actually two curves on this diagram, one for the bouncer in the linear potential and one for a bouncer in the oscillatory potential with $w_{a}=13.10$ and $w_{b}=12.90$. The results overlay perfectly and we can observe no difference at all to the level of accuracy of the calculation. This diagram also displays the collapse and revival phenomena typical of quantum systems and well described by 

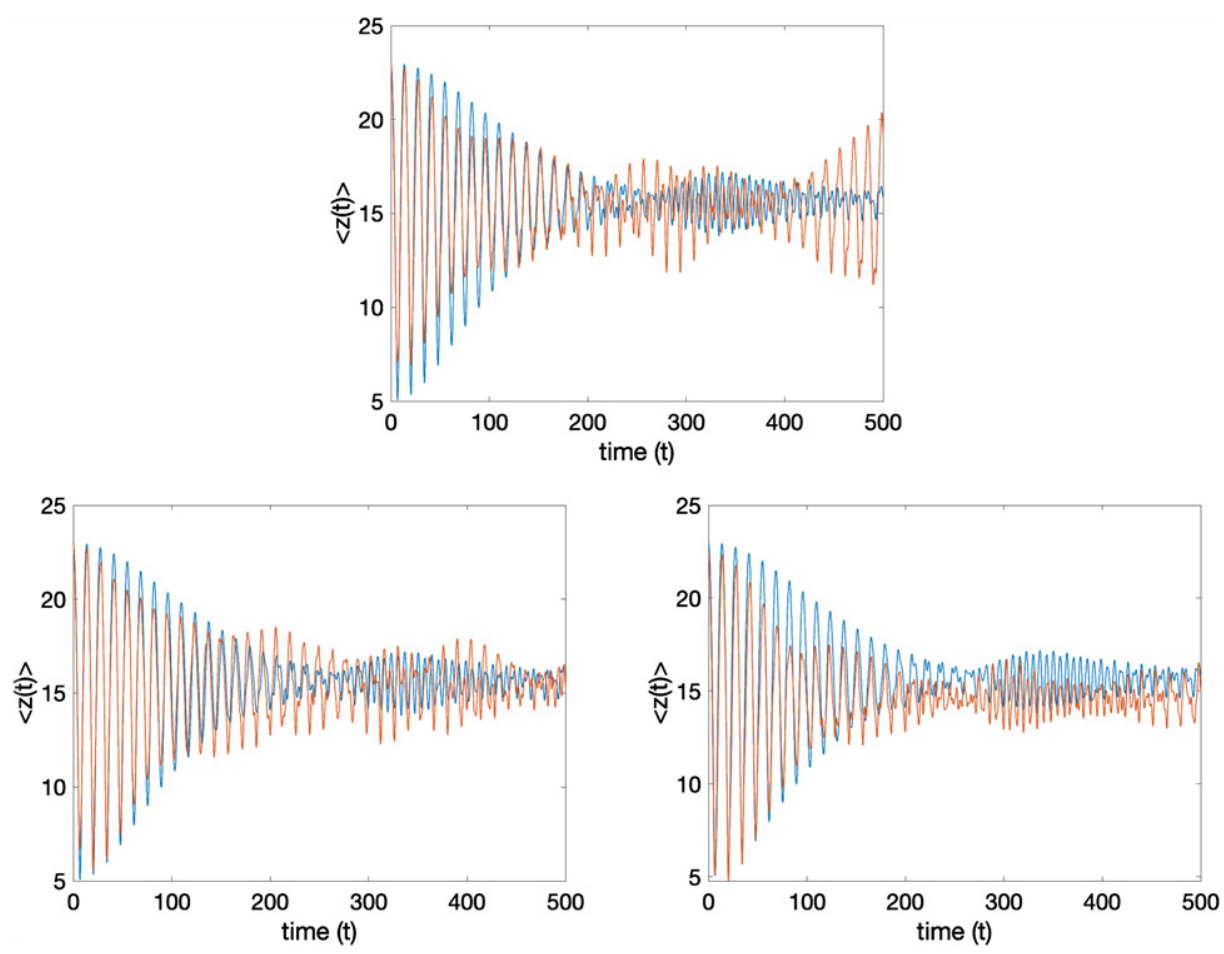

Figure 5. The expectation value of position as a function of time for $z_{0}=23.0$, $\sigma=2.0$. Top: red line for potential with $w_{a}=13.10, w_{b}=12.90$, Lower left: red line for potential with $w_{a}=13.01, w_{b}=12.99$, Lower right: red line for potential with $w_{a}=15.10, w_{b}=14.90$. In each diagram the blue line is the result for the linear potential.

Gea-Banacloche [4]. On the right side we have the expectation value of position as a function of time when the initial drop height is $z(0)=35$. Again this picture contains two lines. The blue line is the bouncer in a linear potential and the red line is a bouncer in the oscillatory potential with $w_{a}=13.10$ and $w_{b}=12.90$. Here the initial height means that we are into the linear regime for the oscillatory potential, but it also means that the contribution to the eigenfunction from the values of $n$ where $w_{n} \approx w_{a}$ are very small. We see that the bounce pattern starts nearly identical but as time increases there is a progressively increasing phase difference between the results of the two potentials and the lines gradually diverge from one another. We find that the higher the initial bounce height the longer it takes for the phase difference to become apparent.

In figure 5 we show the expectation value of position as a function of time for all the potentials shown in figure 1, with the drop height chosen so that some of the large values of $C_{n}$ in the sum in equation (14) have $w_{n} \sim w_{a}, w_{b}$. In each diagram the blue curve is the result for the bouncing particle in the linear potential and the red curve is for an oscillatory potential. The height from which the particle was dropped was $z_{0}=23$ and $\sigma=2$ in all cases. It can be seen that the bounce patterns differ substantially when the key values of $w_{n}$ are close to $w_{a}$ and $w_{b}$.

The results shown in figure 5 show that the behaviour of particles in the oscillatory potentials can be quite different to the behaviour in a linear potential and that, if we could control then potential, it would be possible to use the potential to manipulate the bounce pattern. A question that then arises is can a bouncer in an oscillatory potential exhibit behaviour that is impossible 


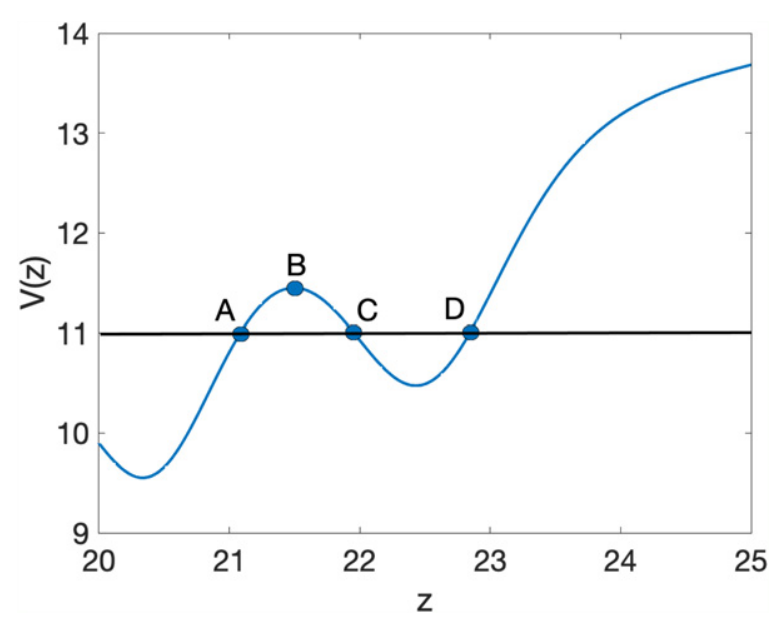

Figure 6. Magnified view of the potential from the upper right diagram of figure 1 . The points $A, B, C$ and $D$ are marked. These are points on the $z$-axis from which we have initiated the bouncing to obtain the density plots of figures 7 and 8 .

for the bouncer in a linear potential. To address this we start by considering figure 6 . Here we have a magnified view of the highest peak in the potential for $w_{a}=13.10$ and $w_{b}=12.90$ shown in the top right picture in figure 1. On this picture we have labelled four values of $z$ from where we will release the bouncer. Points $A, C$ and $D$ all have the same potential energy and point $B$ is at the peak between $A$ and $C$.

We show the space-time map of the probability density for the bouncer in a linear potential in figure 7 and in the oscillatory potential in figure 8 for the different values of the initial height indicated in figure 6. While these figures are superficially similar, there are also some key differences. As with any quantum wavepacket, this one broadens with time and any differences in the wavepackets become washed out in this process, so we look at the first few bounces only. Even so we should keep in mind that the initial wavepacket has a width considerably greater than the wavelength of the potential. We also need to remember that the wavepacket is made up of a large number of eigenfunctions, one of the effects of which is to give it an uncertainty in its energy.

First we consider the particle whose initial position is at point $A$. Classically it would just fall to the ground level starting at $t=0$ and the same thing happens in the linear potential as indicated by the steadily decreasing magnitude of the probability density as we follow the path of the particle. For the oscillatory potential the initial peak is slightly narrower due mainly to the fact that a zero is 'missing' in the oscillatory case. In the linear case there is a time close to the top of each bounce where three eigenstates contribute more or less equally to the wavepacket, while in the oscillatory case it is only two eigenfunctions. After the initial release this diagram shows that, if we measure the height of the bouncer, the probability of finding it close to the minimum below $A$ is very high and the probability of finding it above its initial release height decreases exponentially.

Next consider a particle starting at point $B$. Classically the particle could fall into the local minimum at about $z=22.5$ or it could fall towards the ground. In the linear potential the probability density looks (unsurprisingly) very similar to the probability density for a particle stating at point $A$, the whole diagram has just been moved upwards by the difference in $z$ between point $B$ and point $A$, so the wavepacket will just accelerate downwards. This is not 

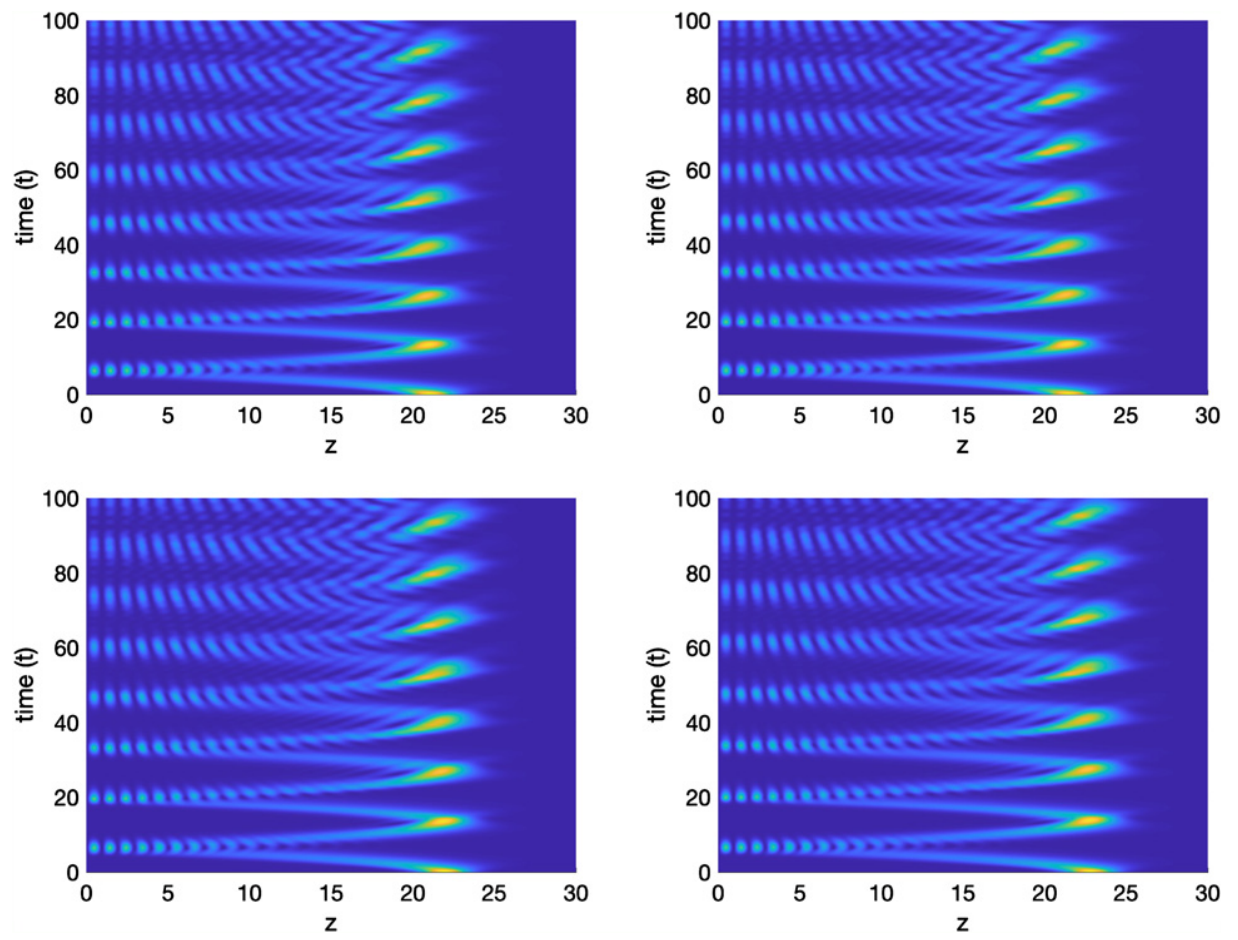

Figure 7. The space-time map for a quantum bouncer for the first few bounces in the linear potential with $\sigma=2.0$ and for initial heights the same as those at points $A$ (top left), $B$ (top right), $C$ (bottom left) and $D$ (bottom right) in figure 6 .

true for the oscillatory potential. Initially the probability density divides into two. It has a peak above $B$ close to the minimum between $C$ and $D$ and another peak close to the minimum below $A$. The wavepacket can fall to the ground directly if it falls to the left of $B$. The probability density shows a series of peaks as a function of time which correspond to the particle slowing down towards the maxima in the potential. The particle can initially fall to the right of $B$ in which case it falls into the local minimum between $C$ and $D$ and can then escape in two ways, Firstly it can go over the peak of the potential at $B$ at a later time because the uncertainty in energy due to the breadth of the wavepacket may give it enough energy to do so. Secondly it can tunnel through the barrier to point $A$ where it will fall to the left. After the first bounce there are two peaks in the probability density which correspond to the same two minima and they correspond to the particle making it over/through the barrier or not. As time increases the wavepacket broadens and the position of the wavepacket becomes increasingly difficult to define. Here we have a key difference between the linear potential and the oscillatory potential. If the particle starts at point $B$ in the oscillatory potential, at a later time it has a substantial probability of being found above the initial drop height.

For a particle that starts from point $C$ in the linear potential the quantum results is again very similar to the probability density for a particle stating at point $A$ in the linear potential, the whole diagram has just been moved upwards by the difference in $z$ between point $C$ and point $A$. For a particle that starts at $C$ the only classical result is for it to fall up to the local minimum at $z \approx 22.5$. Indeed we find that this can happen quantum mechanically as well, the wavepacket can fall upwards for a short distance and the probability density has a peak at this 

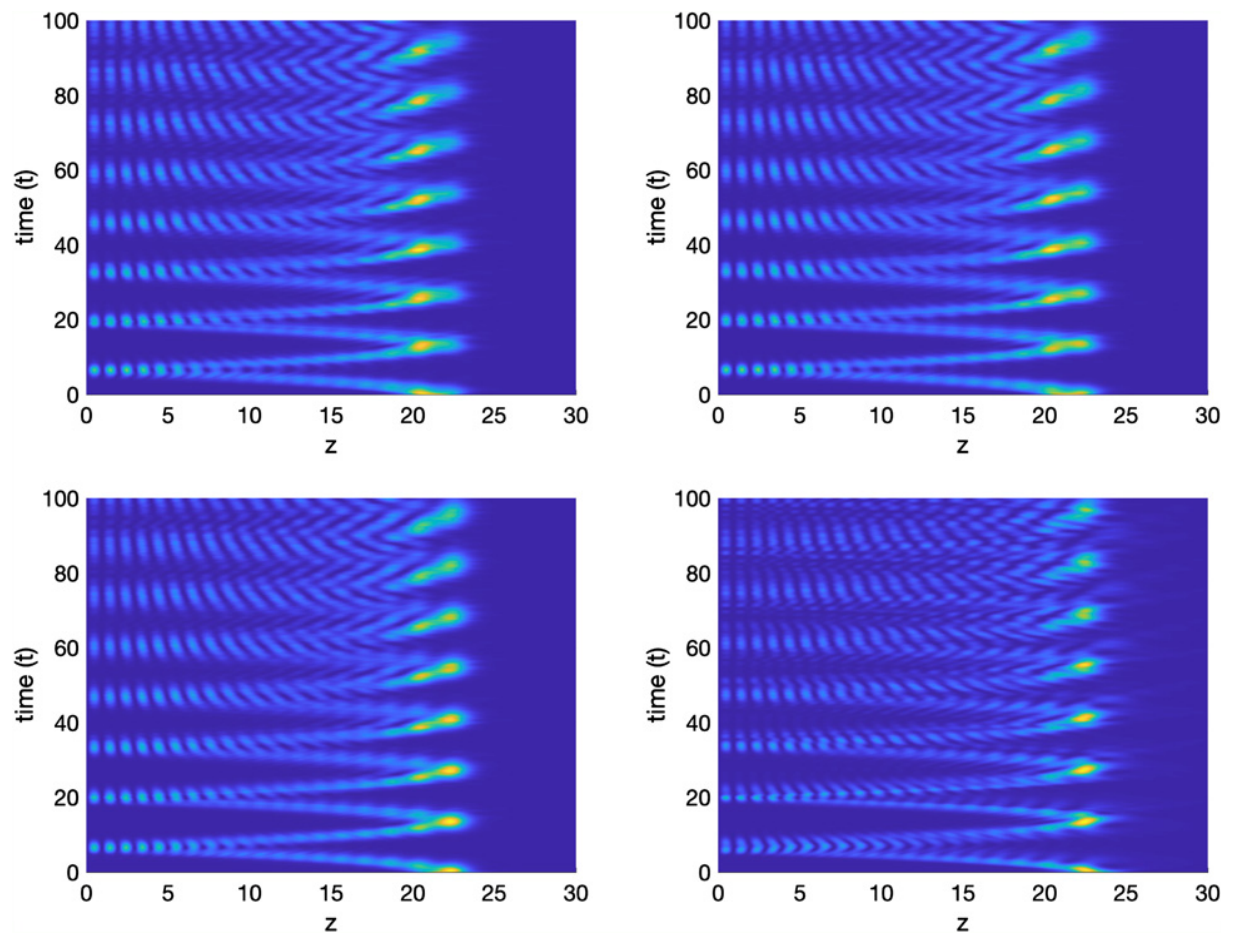

Figure 8. The space-time map for a quantum bouncer for the first few bounces in the oscillatory potential with $\sigma=2.0$ and $w_{1}=13.1$ and $w_{2}=12.9$ for initial heights the same as those at points $A$ (top left), $B$ (top right), $C$ (bottom left) and $D$ (bottom right) indicated in figure 6 .

point which persists for some time. This tells us that the particle has a high probability of being measured at a position above its release point in this case as well. For a particle starting at $C$ the expectation value of energy is substantially greater than that for the particle released at $A$ because, although the potential energy at this point is the same as at point $A$, the whole wavepacket has moved up in energy and the width of the wavepacket is considerably greater than the wavelength of the potential. It can tunnel or get over the potential barrier at $B$ and fall towards the ground. With this greater energy there is also a greater probability of tunnelling back to the minimum between $C$ and $D$ at the top of subsequent bounces. Hence there is again a substantial probability of measuring the particle's position as being above its initial drop height.

A particle starting at $D$ has a greater expectation value of total energy again. It has a strong probability of being trapped in the potential minimum between $C$ and $D$ for a time, but after it escapes from there via tunnelling or just getting over the barrier it has sufficient kinetic energy to overcome all the subsequent barriers with little delay. The probability of measuring the particle above its initial drop height decreases exponentially.

We deduce from this analysis that if we measure the height of the particle at the top of its bounce there is an exponentially small probability of measuring it above its initial drop height for the linear potential, but a very strong probability of measuring it above its initial drop height in the oscillatory potential for some values of the initial drop height. 


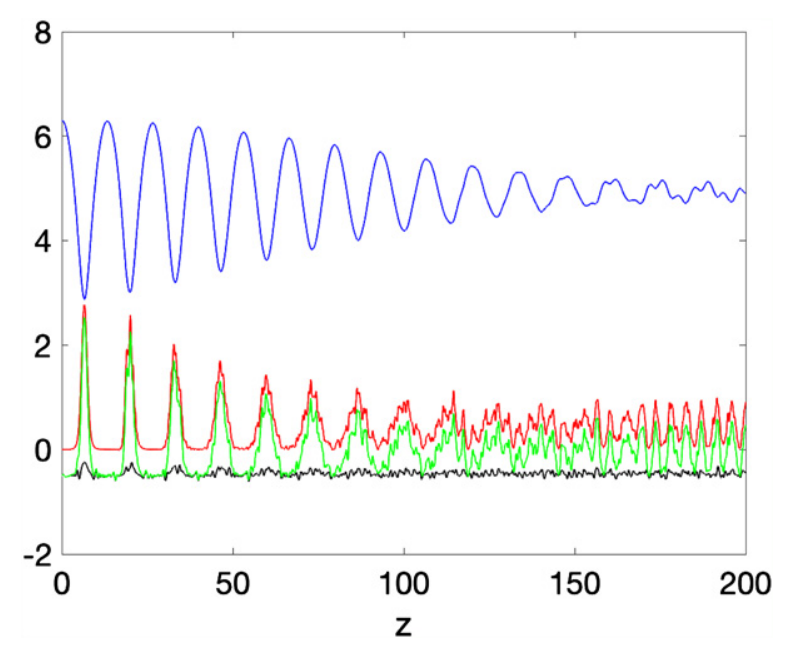

Figure 9. Black line: the expectation value of the negative of the gradient of the potential $\langle-\mathrm{d} V(z) / \mathrm{d} z\rangle>$. Red line: the surface contribution to the rate of change of the expectation value of momentum, i.e. the contribution of the last term in equation (17). The total rate of change of momentum, i.e. the sum of the red and black lines. Blue line: the expectation value of position as a function of time for a wavepacket released from point $B$ in figure 6 . This line has been scaled and is included for reference.

\section{Bouncing}

The expectation value of the position of the wavepacket oscillates and Gea-Banacloche [4] has shown how to get the expectation value of position of the wavepacket in the linear potential to exhibit classical, perfectly elastic bouncing. We can ask the question: why does it bounce? and there are two related answers to this which we discuss below. The first is the most straightforward and well-known. We are adding a large number of functions each of which contains a complex exponential in time. This sum is then multiplied by its complex conjugate to obtain a probability density. In the off-diagonal terms in this multiplication the time dependence will not cancel and the probability density, and hence expectation values, exhibit some sort of harmonic time dependence which does not disappear with time, rather it has a repeat time which is the collapse and revival time discussed by Gea-Banacloche.

The second reason for the bouncing is a purely quantum mechanical force. The potential barrier at ground level is infinite, but we have taken that into account by only allowing eigenfunctions which have $\phi_{n}=0$ at $z=0$. A further purely quantum mechanical force exists when we have an infinite potential. From Ehrenfest's theorem

$$
\frac{\partial\langle p\rangle}{\partial t}=\left\langle\frac{\partial V}{\partial z}\right\rangle+\frac{\hbar^{2}}{2 m}\left[\frac{\partial \Psi}{\partial z} \frac{\partial \Psi^{*}}{\partial z}\right]_{0}
$$

Here the conventional notation is a bit ambiguous. The small square brackets denote a commutator and the large ones indicate that the quantity inside them should be evaluated at the position indicated ( $z=0$ in this case). So as well as the usual term dependent on the gradient of the potential there exists a further force dependent on the gradient of the wavefunction at the surface. In figure 9 we illustrate the effect of this force. The black line here is the quantum equivalent of the usual force felt by a particle, i.e. it is the expectation value of the gradient of the potential. As expected, this is always negative, pulling the wavepacket towards the ground. 
The red line is the second term in equation (17), it is a contribution to the rate of change of the expectation value of momentum from the surface and it is always non-negative. The green line is the total rate of change of expectation value of momentum calculated both directly and as the sum of the two terms on the right-hand side of equation (17). They are identical. We have also calculated this quantity (force) as $m \mathrm{~d}^{2}\langle\hat{x}\rangle / \mathrm{d} t^{2}$ and obtained the same result. The blue line is the expectation value of position for a particle dropped from point $B$ although this line has been scaled down by a factor of eight and moved upwards as it is presented here only for comparison with the other lines. We see from this diagram that for most of the bounce, particularly close to the top of the bounce, the particle feels the rate of change of potential, but not the surface force. However close to the bottom of the bounce the surface term dominates so the total force changes sign and we end up with a force upwards which will assist with the bouncing.

\section{Discussion}

Employing time-dependent supersymmetry we have found a family potential and eigenfunctions that describes a particle in an oscillatory gravitational field. The potential contains two dimensionless arbitrary parameters $w_{1}$ and $w_{2}$ which enable us to control the oscillation wavelength and amplitude. When these two parameters differ substantially the potential contains infinities which are of interest, but which are not the focus of this paper. If $\left|w_{1}-w_{2}\right|<0.3$ we find a finite oscillatory gravitational potential in which a wavepacket exhibits bouncing behaviour. The bouncing is very much unlike classical bouncing in which the bouncer accelerates downwards until it hits the ground. In this case the motion is due to the interference of wavefunctions and the particle initially accelerates, but then decelerates and reversing its motion before reaching the ground. We have pointed out both the similarities and the differences of motion in the oscillatory potential compared with the usual linear bouncer. In particular we have indicated how we might observe that the field is oscillatory, namely that at some drop heights it is possible that a subsequent measurement of the height of the bouncer will yield an answer that is substantially higher than the initial drop height. Because we have not found it mentioned in previous papers on the quantum bouncer we have highlighted the 'quantum force' which always occurs in quantum mechanics when we have an infinite potential, and discussed its role in bouncing.

We have plotted both the expectation value of position in figures 4 and 5 and probability density maps in figures 7 and 8 . The path of the particle as measured from the expectation values looks very different from the path as indicated by the probability density. This leads us to question how useful the expectation value is for a system with an infinite potential. In this case the wavefunction is only non-zero above $z=0$ because of the infinite potential and the fact that the eigenfunctions have to go to zero at the point where the potential becomes infinite. This means all the weight of then probability density is above zero and the expectation value is mathematically prohibited from getting close to zero, while the probability density, not being an integrated quantity, can get arbitrarily close to $z=0$.

The work reported in this paper is the result of a student project. The student was required to study some mathematical research papers in detail which enhances both their research skills and understanding of the subject. The students also get to experience standard mathematical software such as Mathematica or Maple. Furthermore they have to perform some original mathematics in calculating observables and then interpret it intelligently. This work clearly demonstrates that time-dependent supersymmetry provides an excellent starting point for a theoretical physics project. 


\section{ORCID iDs}

P Strange (1) https://orcid.org/0000-0001-5818-8032

\section{References}

[1] Sakurai J J 1994 Modern Quantum Mechanics (Reading, MA: Addison-Wesley)

[2] Merzbacher E 1998 Quantum Mechanics 3rd edn (New York: Wiley)

[3] Gibbs R L 1975 Am. J. Phys. 43 25-8

[4] Gea-Banacloche J 1999 Am. J. Phys. 67776

[5] Lekner J and Nguyen H 2009 Eur. J. Phys. 30 L67

[6] Chaterjee R 2019 arXiv:1905.11220v2[quant-ph]

[7] Mather W H and Fox R F 2006 Phys. Rev. A 73032109

[8] Wheeler N 2003 Reed College,

[9] Doncheski M A and Robinett R W 1999 Eur. J. Phys. 20 29-37

[10] Nevizhevsky V V et al 2002 Nature 415 297-9

[11] Nevizhevsky V V et al 2003 Phys. Rev. D 67102002

[12] Jenke T, Geltenbort P P, Lemmel H and Abele H 2011 Nat. Phys. 7 468-72

[13] Abele H, Cronenberg G, Geltenbort P, Jenke T, Lins T and Saul H 2011 Physics Procedia 17 4-9

[14] Abele H and Leeb H 2012 New J. Phys. 14055010

[15] Bagrov V G, Samsonov B F and Shekoyan L A 1995 Russian Physics Journal 38 706-12

[16] Bagrov V G and Samsonov B F 1996 Phys. Lett. A 210 60-4

[17] Bagrov V G and Samsonov B F 1997 arXiv:quant-ph/9709040v1

[18] Schulze-Halberg A and Carballo-Jimenez J M 2009 Symmetry 1 115-44

[19] Cooper F, Khare A and Sukhatme U 2001 Supersymmetry in Quantum Mechanics (Singapore: World Scientific)

[20] Zelaya K and Rosas-Ortiz O 2017 J. Phys.: Conf. Ser. 839012018

[21] Rasinskaite D and Strange P 2020 Eur. J. Phys. accepted

[22] Abramowitz M and Stegun I A 1972 (New York: Dover) 